\title{
miR-141 and miR-375 induction and release are different from PSA mRNA and PCA3 upon androgen stimulation of LNCaP cells
}

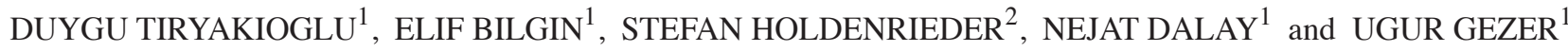 \\ ${ }^{1}$ Department of Basic Oncology, Institute of Oncology, Istanbul University, Capa, Istanbul 34390, Turkey; \\ ${ }^{2}$ Institute of Clinical Chemistry and Pharmacology, University of Bonn, D-53105 Bonn, Germany
}

Received May 16, 2013; Accepted July 10, 2013

DOI: $10.3892 /$ br.2013.135

\begin{abstract}
Recent studies have demonstrated the differential expression of miR-141 and miR-375 in circulation of patients with advanced/metastatic prostate cancer (PCa). The aim of this study was to investigate the regulation of miR-141 and miR-375 by androgens and their release into the incubation medium in relation to prostate-specific antigen (PSA) mRNA and prostate cancer antigen 3 (PCA3). Plasma levels of these molecules were measured in a small cohort of patients with localized PCa. As an in vitro cell model we used androgen-sensitive LNCaP cells exposed to an androgen ablation of $48 \mathrm{~h}$, and then treated with dihydrotestosterone (DHT) for $24 \mathrm{~h}$. Expression of the four RNA molecules was measured by quantitative polymerase chain reaction (qPCR). miR-141 and miR-375 were induced in a dose-dependent manner where the median stimulation reached only 1.5-fold at maximum. The effect of DHT on PSA mRNA (up to 30-fold) and PCA3 (up to 195-fold) was much more evident. With regard to the release into the incubation medium, similar results were obtained with the exception of PCA3. At the highest DHT dose (100 nM), median miR-141 and miR-375 release was increased 1.7 - and 1.4-fold $(\mathrm{P}=0.07)$, respectively. DHT treatment led to a significant release of PSA mRNA (up to 12 -fold) into the medium while PCA3 could not be amplified from the incubation medium. In plasma only PCA3 differed significantly between localized PCa patients and healthy subjects. In conclusion, our study provides evidence that miR-141 and miR-375 are increasingly released into incubation medium from androgen-stimulated cells. However, the extent of their induction was weaker than PSA mRNA or PCA3, suggesting differential regulation by androgens.
\end{abstract}

\section{Introduction}

Prostate cancer $(\mathrm{PCa})$ is one of the most prevalent malignant diseases among men in the Western countries (1). Early stage

Correspondence to: Professor Ugur Gezer, Department of Basic Oncology, Institute of Oncology, Capa, Istanbul 34390, Turkey

E-mail: ugurd@istanbul.edu.tr

Key words: prostate cancer, androgens, microRNAs, molecule release
PCa depends on androgen for growth (2). The most effective systemic treatment for this hormone-sensitive cancer has been androgen deprivation therapy. In many cases, however, after an initial regression the tumor evolves to castration resistant prostate cancer (CRPC), which is defined as the emergence of tumor growth in the presence of castrating levels of androgens, defined as serum testosterone $50 \mathrm{ng} / \mathrm{ml}$ and the growth of measurable disease. The serum prostate-specific antigen (PSA) test along with the digital rectal examination has been used for the detection of early stage PCa. The PSA test is also routinely used to monitor PCa recurrence after therapy. However, it has limited accuracy in predicting treatment outcomes and in decision making in the clinic (3) necessitating additional prognostic markers for PCa.

A previous study revealed that circulating microRNAs in the blood of cancer patients deserve particular attention as potential diagnostic and prognostic biomarkers (4). miRNAs are present in human plasma in a stable form that is protected from endogenous RNase activity (4) as most of the circulating miRNAs are included in lipid or lipoprotein complexes, such as apoptotic bodies, microvesicles or exosomes (5). Comprehensive screening of serum or plasma miRNAs by quantitative polymerase chain reaction (qPCR) or microarray technologies, or the quantification of single miRNAs in distinct PCa patient cohorts and healthy individuals have been reported in several studies (for a review see ref. 6). However, the overlap of relevant miRNA types between different studies is small. Discrepancies are likely due to different miRNA platforms, quantification protocols, cut-off levels, patient selection and small cohort sizes. However, two promising miRNAs, miR-141 and miR-375, have emerged as circulating diagnostic and prognostic markers across independent studies (7-10). A recent study also showed that among others, miR-141 is androgen-regulated in cell lines and xenografts (11) and modulates transcriptional activity of the androgen receptor (AR) in human PCa cells by targeting the small heterodimer partner protein, a co-repressor to AR (12). In this study, we hypothesized that if miR-141 and eventually miR375 are androgen-regulated, following dihydrotestosterone (DHT) treatment of androgen-sensitive LNCaP cells, an increase in expression would lead to a higher release into the incubation medium mimicking in vivo conditions. In this context, we also analyzed prostate-specific antigen mRNA (PSA mRNA) and PCa antigen 3 (PCA3) expression levels. PCA3 appears to be an appropriate control molecule 
as the expression of this non-coding RNA is restricted to cell lines that express AR, such as LNCaP cells (13). It has recently been shown that PCA3 is induced by DHT and modulates the expression of AR target genes (14). In order to compare the levels of these molecules in culture medium with those in blood circulation, their plasma levels were also measured in a small cohort of patients with localized PCa.

\section{Materials and methods}

Culture and hormone treatment of LNCaP cells. Human prostate adenocarcinoma LNCaP cells were originally obtained from the American Type Culture Collection (Manassas, VA, USA). LNCaP cells were routinely maintained in the regular medium, i.e., RPMI-1640 medium supplemented with $10 \%$ FBS, $1 \%$ glutamine and penicillin/streptomycin. For experiments, androgen-sensitive LNCaP cells with low passage numbers were utilized. Cells were seeded at a density of $1 \times 10^{5}$ cells per well in six-well culture plates and grown for $48 \mathrm{~h}$ in a medium supplemented with $10 \%$ FBS which was treated with dextran-coated charcoal to ablate steroid hormones. The medium was replaced by fresh medium containing DHT (DHT, $\mathrm{C}_{19} \mathrm{H}_{30} \mathrm{O}_{2}$ ) (Sigma-Aldrich, Zwijndrecht, The Netherlands) at concentrations of $0,1,10$ or $100 \mathrm{nM}$, and the cells were allowed to grow for a further $24 \mathrm{~h}$. The cells and media were then harvested and stored for subsequent analysis.

Patient samples. In addition to cell culture experiments we measured miR-141, miR-375, PSA mRNa and PCA-3 in the plasma of localized PCa patients, which was analog to androgen-responsive LNCaP cells. A cohort of 34 patients with pathologically confirmed localized PCa and 15 healthy male individuals were enrolled in the present study. The median age of the patients was 67 . The study was approved by the local ethics committee of the Istanbul Medical Faculty. Peripheral blood was drawn, plasma was separated immediately and stored at $-80^{\circ} \mathrm{C}$ for subsequent analyses. Informed consent was obtained from each patient.

RNA extraction and $q P C R$. RNA molecules were measured from the total RNA fraction of cellular nuclear RNA, culture medium or blood plasma. To isolate total RNA a monophasic phenol and guanidine thiocyanate solution was used (Roche, Mannheim, Germany) according to the manufacturer's protocol. Two hundred microliters of the culture medium were used. Isolated RNA was dissolved in PCR-grade water. cDNA was produced using the miScript II RT Kit (Qiagen, Valencia, CA, USA) which simultaneously converts all RNA species into cDNA. Therefore, miRNAs, PSA mRNa and PCA3 were amplified from the same template. To quantify miRNAs we used miScript Primer Assays (Qiagen) which includes a universal primer specific to the poly-A tail of the miRNA added by poly(A) polymerase and a miRNA-specific primer. SYBR-Green (Qiagen) was used as the fluorescent molecule. The amplified PCR product had a size of $\sim 80 \mathrm{bp}$. The miR-16 molecule was used as an internal control as it exhibited a relatively stable expression during androgen treatment through various doses in LNCaP cells and in blood plasma. GAPDH was used as the internal control for PSA mRNA and PCA3. Real-time PCR was performed using the LightCycler 480 Instrument (Roche).
Amplification of the appropriate product for each molecule was confirmed by melting curve analysis. Expression levels of each RNA were originally presented as the threshold cycle $(\mathrm{Ct})$ values, defined as the fractional cycle number at which the fluorescent signal exceeds the fixed threshold in qRT-PCR. For data analysis, we used the comparative $\mathrm{Ct}$ method $(\mathrm{ct} t)$, normalized by subtracting the $\mathrm{Ct}$ value of endogenous reference from each RNA. Data were then summarized as the mean of $\mathrm{cCt}$ value for each RNA.

Statistical analysis. This study tested the hypothesis that miR-141 and miR375 are androgen-regulated in a similar manner to PSA and PCA3 and, that induction by DHT would lead to elevated levels of release from LNCaP cells. For this purpose, at least five independent cell culture experiments were performed to evaluate the expression and release of RNA molecules. Changes relative to basal levels were expressed as 'fold changes' and median values were statistically compared by the median test. The Jonckheere-Terpstra trend test was used to assess the distribution of the RNA molecules over DHT doses. To analyze correlations between the molecules we utilized the Pearson correlation test while differences were assessed using the Mann-Whitney $\mathrm{U}$ test. $\mathrm{P}<0.05$ was considered as the level of significance.

\section{Results}

Androgen regulation of $m i R-141, m i R-375, P S A m R N a$ and PCA3. When basal levels of miR-141 and miR-375 in LNCaP cells were compared, we observed that miR-141 is expressed (mean relative value 0.14 ) at much higher levels than miR-375 (mean relative value 0.005). Basal levels of PCA3 were extremely low and its expression was $\sim 12,500$-fold lower than that of PSA mRNA (mean relative value 0.07). In hormone-treated cells, changes relative to basal levels were expressed as 'fold changes'. As seen in Fig. 1, the effect of DHT on the expression of four molecules is variable. Both miRNAs were weakly induced in a dose-dependent manner where the median stimulation reaches only 1.32-fold for miR-141 $(\mathrm{P}=0.07)$ and 1.5-fold for miR-375 ( $\mathrm{P}=0.07)$ at maximum (Fig. 1A and B). The P-values for the distribution of miR-141 and miR-375 expression over DHT doses were 0.057 and 0.033 , respectively (Jonckheere-Terpstra trend test). In contrast to miRNAs, the effect of DHT on PSA mRNA and PCA3 was much more evident (Fig. 1C and D). The two molecules were significantly upregulated in a dose-dependent manner $(\mathrm{P}<0.001$ for their distributions). The median induction of PSA mRNA was 14- to 30-fold while the effect was much more pronounced for PCA3 (71- to 195-fold). Of note, the miR-141 expression in LNCaP cells, but not miR-375 expression, was correlated with PSA mRNA and PCA3 ( $\mathrm{P}<0.01$ for both comparisons).

Kinetic release of $m i R-141, m i R-375, P S A$ mRNa from LNCaP cells. With regard to the release into the incubation medium, similar results were observed, with the exception of PCA3. In the culture medium, PCA3 was below the limit of detection due to low levels of basal expression in cells despite up to 400-fold induction. At the highest DHT dose (100 nM), median miR-141 and miR-375 releases were increased 1.7- and 1.4-fold $(\mathrm{P}=0.07)$, respectively (Fig. 2A and B). As expected, DHT treatment led to 


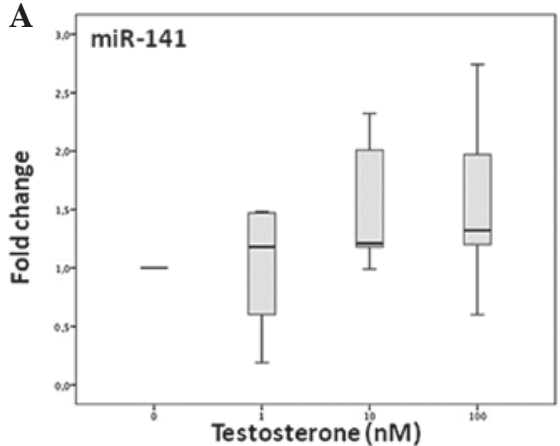

C

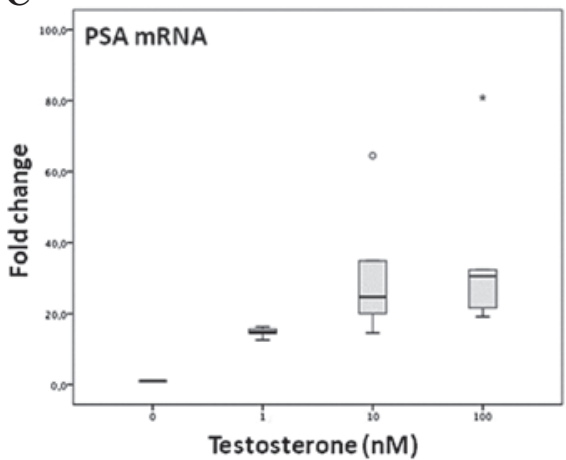

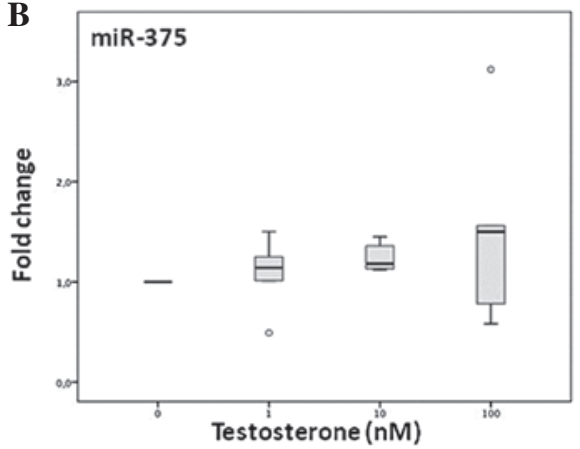

D

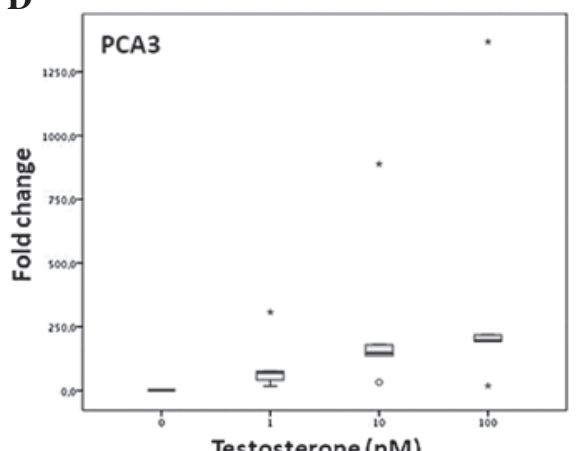

Figure 1. Androgen regulation of miR-141, miR-375, prostate-specific antigen (PSA) mRNa and prostate cancer antigen 3 (PCA3). Following hormone depletion for $48 \mathrm{~h} \mathrm{LNCaP}$ cells were treated with $0,1,10$, and $100 \mathrm{nM}$ dihydrotestosterone (DHT) for $24 \mathrm{~h}$ and RNA molecules quantified. miR-16 was used as the reference gene for miR-141, miR-375, while GAPDH was the reference gene for PSA mRNa and PCA3. Results of five were experiments were evaluated. Basal level (0 nM DHT) of each molecule was taken as 1 , and changes expressed as 'fold changes'. Box plots show median value, minimum and maximum values and quartiles.
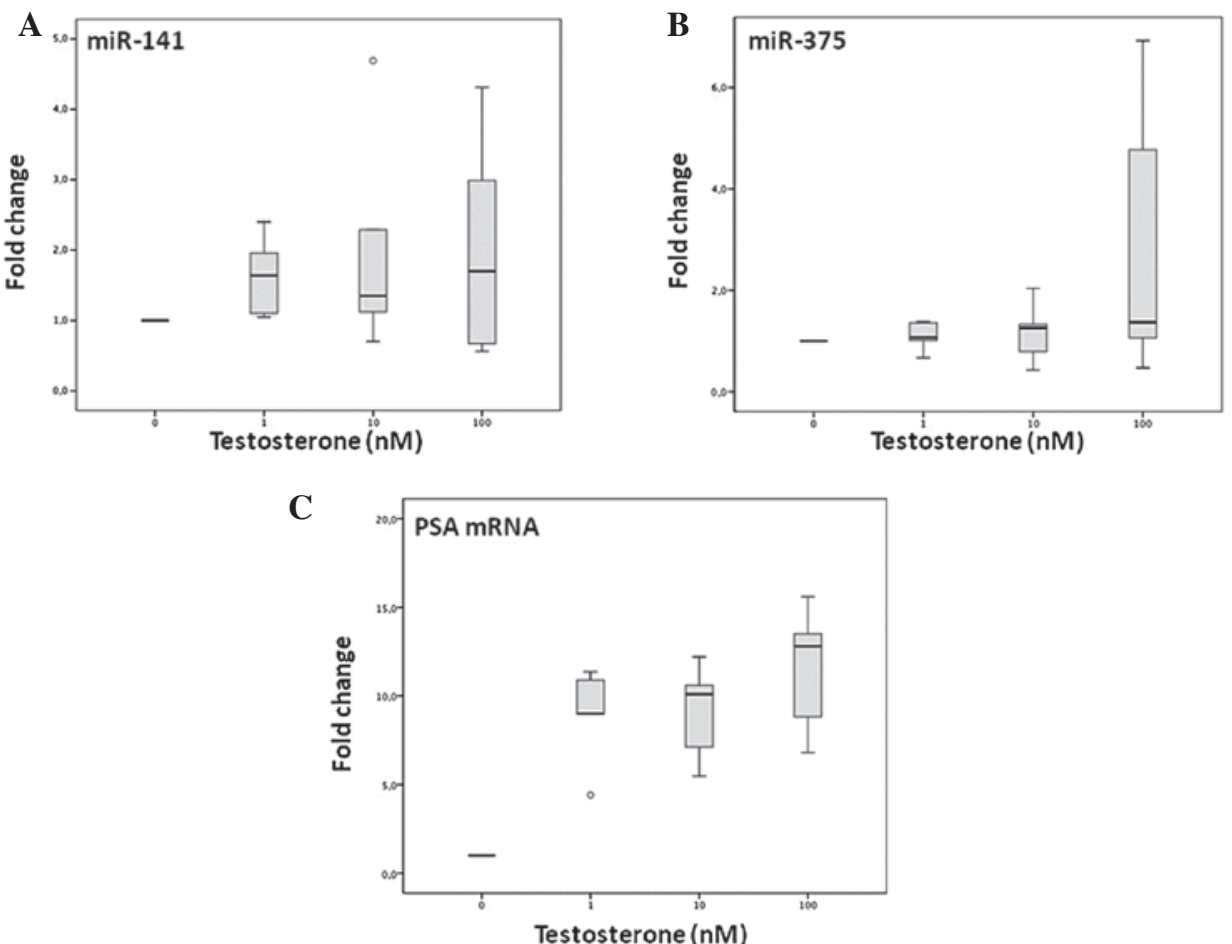

Figure 2. Kinetic release of miR-141, miR-375, prostate-specific antigen (PSA) mRNa from LNCaP cells. RNA molecules were measured from the culture media of cells treated with dihydrotestosterone (DHT) as described above.

a high release of PSA mRNA (9- to 12-fold) (Fig. 2C). Medium levels of PSA mRNA correlated to its expression in LNCaP cells $(\mathrm{P}=0.005)$ while this did not apply to miRNA molecules.
Plasma levels of miR-141, miR-375, prostate-specific antigen (PSA) $m R N a$ and prostate cancer antigen 3 (PCA3) in prostate cancer (PCa) patients. We then analyzed the plasma 

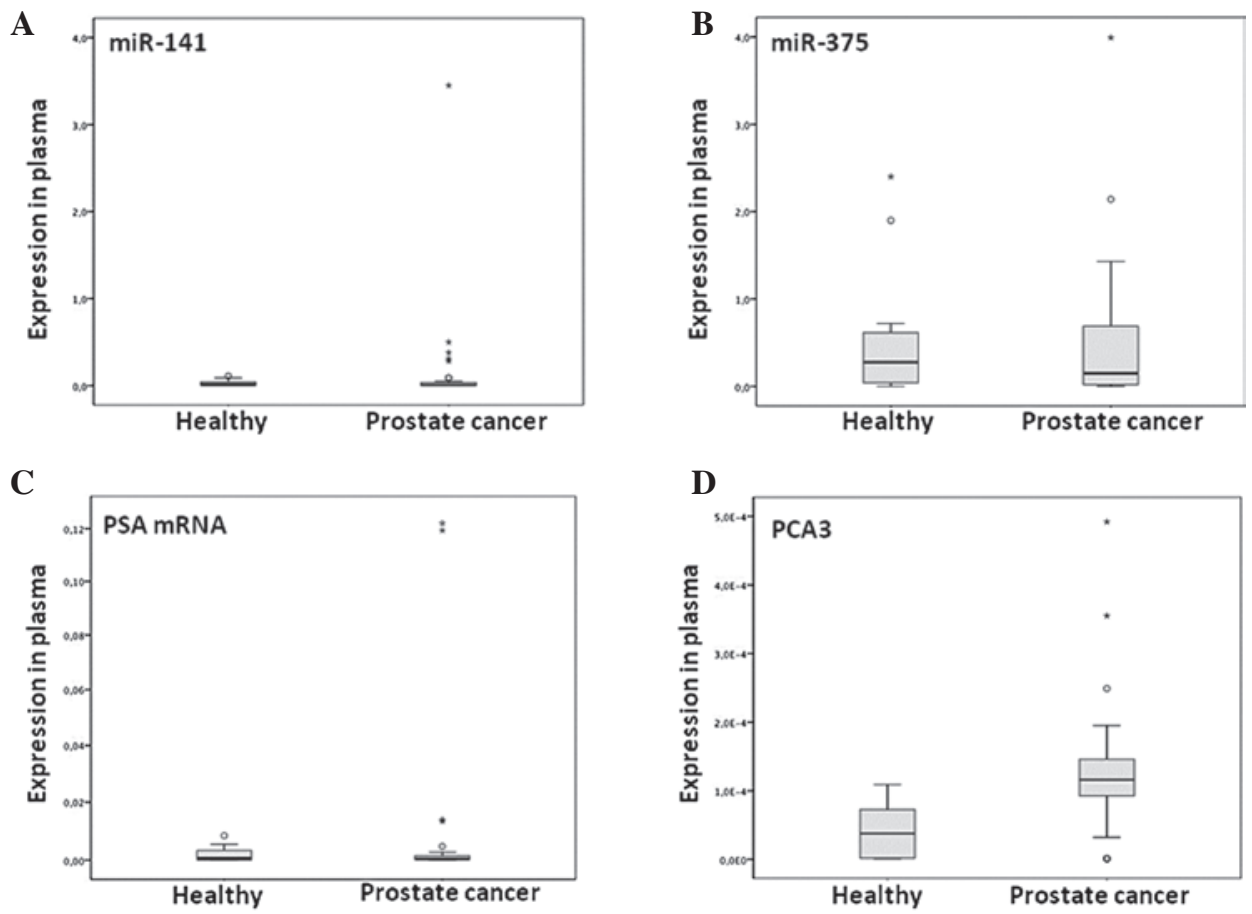

Figure 3. Plasma levels of miR-141, miR-375, prostate-specific antigen (PSA) mRNa and prostate cancer antigen 3 (PCA3) in prostate cancer (PCa) patients. Total RNA was isolated from plasma, and following cDNA synthesis, each RNA molecules was relatively quantified as described above. Outlier values are also shown.

levels of these molecules in PCa patients and healthy subjects. Unlike LNCaP cells miR-375 exhibited higher plasma levels compared with miR-141 in the study group (median relative values 0.15 and 0.006 , respectively, $\mathrm{P}<0.001$ ) (Fig. $3 \mathrm{~A}$ and $\mathrm{B}$ ). There was no linear correlation between the two molecules $(\mathrm{P}=0.6)$ and their levels did not statistically differ between the patients and the control samples $(\mathrm{P}>0.05)$. Similarly, circulating PSA mRNA levels were similar between the study groups and did not correlate with serum PSA protein $(\mathrm{P}>0.05)$. PCA3 was present in low amounts in plasma with many samples harboring undetectable amounts of PCA3 (Fig. 3D). However, significantly higher levels of PCA3 were detected in some plasma samples $(\mathrm{P}=0.001)$.

\section{Discussion}

Recent studies (7-10) have identified the differential expression of miR-141 and/or miR-375 in circulation of patients with advanced PCa or metastatic CRPC. miR-141 has also been shown to predict clinical PCa progression when compared with other clinically validated biomarkers such as PSA or circulating tumor cells (15). Results of a recent study have shown that miR-141 is androgen-regulated (11) and that it can modulate AR transcriptional activity in human PCa cells (12). The aim of the present study was to assess the release of these miRNAs into the incubation medium from DHT-induced PCa cells. To the best of our knowledge, this study is the first to experimentally evaluate miR-141 and miR-375 release from androgen-stimulated cells and correlate them with PSA mRNA and PCA3.

Our results show that DHT differentially affects the expression of miR-141, miR-375, PSA mRNA and PCA3 in LNCaP cells. When compared to the markedly stimulated
PSA mRNA and PCA3 expression, DHT treatment weakly induced miR-141 and miR-375 expression (up to 1.5-fold). Waltering et al (11) reported a very similar rate for miR-141. Of note, miR-141 expression in LNCaP cells, but not miR-375 expression, was correlated with PSA mRNA and PCA3. Nevertheless, our findings suggest that miR-375 may be regulated in a similar manner as miR-141 by androgens. However, the regulation of the two miRNAs by androgen is weaker than PSA or PCA3, which were specifically induced by DHT. Considering for example, the prostate-specific PCA3 expression (13) and its role in the modulation of AR target genes (14), PCA3 appears to be an essential component of AR signaling which may require higher levels of stimulation than miR-141. In line with this, unlike miRNAs, even a small dose of DHT $(1 \mathrm{mM})$ was effective to strongly induce PCA3 or PSA mRNA. Furthermore, we found significant higher plasma levels of PCA3 in PCa patients than in healthy subjects indicating its specificity for prostate. The presence of PCA3 in blood has also been described recently (16). Plasma levels of miR-141, miR-375 and PSA mRNA in PCa did not significantly differ from those in healthy subjects, suggesting that these molecules are not increased in circulation of patients with local disease.

The main objective of the study was to characterize the release of miR-141 and miR-375 from androgen-stimulated cells. Similar to their expression in the LNCaP cells, miR-141 and miR-375 were released in a limited quantity into the culture medium (up to 1.7-fold). This was a small change when compared with PSA mRNA which is released in much higher amounts (up to 11-fold) as has been previously reported for PSA subsequent to androgen stimulation (17). Nevertheless, an increased release of miR-141 and miR-375 from androgen-stimulated cells may explain their higher expression in the blood of patients with advanced PCa or metastatic CRPC (7-10) in 
which, in addition to androgen production by the adrenal gland and testis, several of the enzymes involved in the synthesis of androgens are highly expressed in tumor tissue (18).

In conclusion, our study provides evidence that miR-141 and miR-375 are induced and increasingly released into incubation medium from androgen-stimulated LNCaP cells. However, the extent of induction and release was weaker than that of PSA mRNA or PCA3 suggesting differential regulation by androgens.

\section{Acknowledgements}

This study is part of the MSc thesis of Duygu Tiryakioğlu and was supported by the Scientific Research Coordination Unit of Istanbul University (Project nos. 21165 and 28508).

\section{References}

1. Hayat MJ, Howlader N, Reichman ME and Edwards BK: Cancer statistics, trends, and multiple primary cancer analyses from the Surveillance, Epidemiology, and End Results (SEER) Program. Oncologist 12: 20-37, 2007.

2. Leo S, Accettura C and Lorusso V: Castration-resistant prostate cancer: targeted therapies. Chemotherapy 57: 115-127, 2011.

3. Shariat SF, Scherr DS, Gupta A, Bianco FJ Jr, Karakiewicz PI, Zeltser IS, Samadi DB and Akhavan A: Emerging biomarkers for prostate cancer diagnosis, staging, and prognosis. Arch Esp Urol 64: 681-694, 2011.

4. Mitchell PS, Parkin RK, Kroh EM, Fritz BR, Wyman SK, Pogosova-Agadjanyan EL, Peterson A, Noteboom J, O'Briant KC, Allen A, Lin DW, Urban N, Drescher CW, Knudsen BS, Stirewalt DL, Gentleman R, Vessella RL, Nelson PS, Martin DB and Tewari M: Circulating microRNAs as stable blood-based markers for cancer detection. Proc Natl Acad Sci USA 105: 10513-10518, 2008.

5. Kosaka N, Iguchi $\mathrm{H}$ and Ochiya T: Circulating microRNA in body fluid: a new potential biomarker for cancer diagnosis and prognosis. Cancer Sci 101: 2087-2092, 2010.

6. Kuner R, Brase JC, Sültmann H and Wuttig D: microRNA biomarkers in body fluids of prostate cancer patients. Methods 59: 132-137, 2013.

7. Yaman Agaoglu F, Kovancilar M, Dizdar Y, Darendeliler E, Holdenrieder S, Dalay N and Gezer U: Investigation of miR-21, miR-141, and miR-221 in blood circulation of patients with prostate cancer. Tumor Biol 32: 583-588, 2011.
8. Brase JC, Johannes M, Schlomm T, Fälth M, Haese A, Steuber T, Beissbarth T, Kuner R and Sültmann H: Circulating miRNAs are correlated with tumor progression in prostate cancer. Int $\mathrm{J}$ Cancer 128: 608-616, 2011

9. Bryant RJ, Pawlowski T, Catto JW, Marsden G, Vessella RL, Rhees B, Kuslich C, Visakorpi T and Hamdy FC: Changes in circulating microRNA levels associated with prostate cancer. $\mathrm{Br}$ J Cancer 106: 768-774, 2012.

10. Nguyen HC, Xie W, Yang M, Hsieh CL, Drouin S, Lee GS and Kantoff PW: Expression differences of circulating microRNAs in metastatic castration resistant prostate cancer and low-risk, localized prostate cancer. Prostate 73: 346-354, 2013.

11. Waltering KK, Porkka KP, Jalava SE, Urbanucci A, Kohonen PJ, Latonen LM, Kallioniemi OP, Jenster G and Visakorpi T: Androgen regulation of microRNAs in prostate cancer. Prostate 71: 604-614, 2011.

12. Xiao J, Gong AY, Eischeid AN, Chen D, Deng C, Young CY and Chen XM: miR-141 modulates androgen receptor transcriptional activity in human prostate cancer cells through targeting the small heterodimer partner protein. Prostate 72: 1514-1522, 2012.

13. van Bokhoven A, Varella-Garcia M, Korch C, Johannes WU, Smith EE, Miller HL, Nordeen SK, Miller GJ and Lucia MS: Molecular characterization of human prostate carcinoma cell lines. Prostate 57: 205-225, 2003.

14. Ferreira LB, Palumbo A, de Mello KD, Sternberg C, Caetano MS de Oliveira FL, Neves AF, Nasciutti LE, Goulart LR and Gimba ER: PCA3 noncoding RNA is involved in the control of prostate-cancer cell survival and modulates androgen receptor signaling. BMC Cancer 12: 507, 2012.

15. Gonzales JC, Fink LM, Goodman OB Jr, Symanowski JT, Vogelzang NJ and Ward DC: Comparison of circulating microRNA-141 to circulating tumor cells, lactate dehydrogenase and prostate-specific antigen for determining treatment response in patients with metastatic prostate cancer. Clin Genitourin Cancer 9: 39-45, 2011.

16. Neves AF, Dias-Oliveira JD, Araújo TG, Marangoni K and Goulart LR: Prostate cancer antigen 3 (PCA3) RNA detection in blood and tissue samples for prostate cancer diagnosis. Clin Chem Lab Med 51: 881-887, 2012

17. Sun XY, Plouzek CA, Henry JP, Wang TT and Phang JM: Increased UDP-glucuronosyltransferase activity and decreased prostate specific antigen production by biochanin A in prostate cancer cells. Cancer Res 58: 2379-2384, 1998.

18. Montgomery RB, Mostaghel EA, Vessella R, Hess DL, Kalhorn TF, Higano CS, True LD and Nelson PS: Maintenance of intratumoral androgens in metastatic prostate cancer: a mechanism for castration-resistant tumor growth. Cancer Res 68 : 4447-4454, 2008. 